\title{
Linear Dichroism and Resonant Photoemission in Gd
}

\author{
S. R Mishra \\ T. R. Cummins \\ G D Waddill \\ W J Gammon \\ G. van der Laan \\ K W. Goodman \\ $\mathrm{J}$ G Tobin
}

This paper was prepared for submittal to the

Magnetics Multilayer (MML) Conference

Vancouver, CANADA

June 14-19, 1998

May 13, 1998

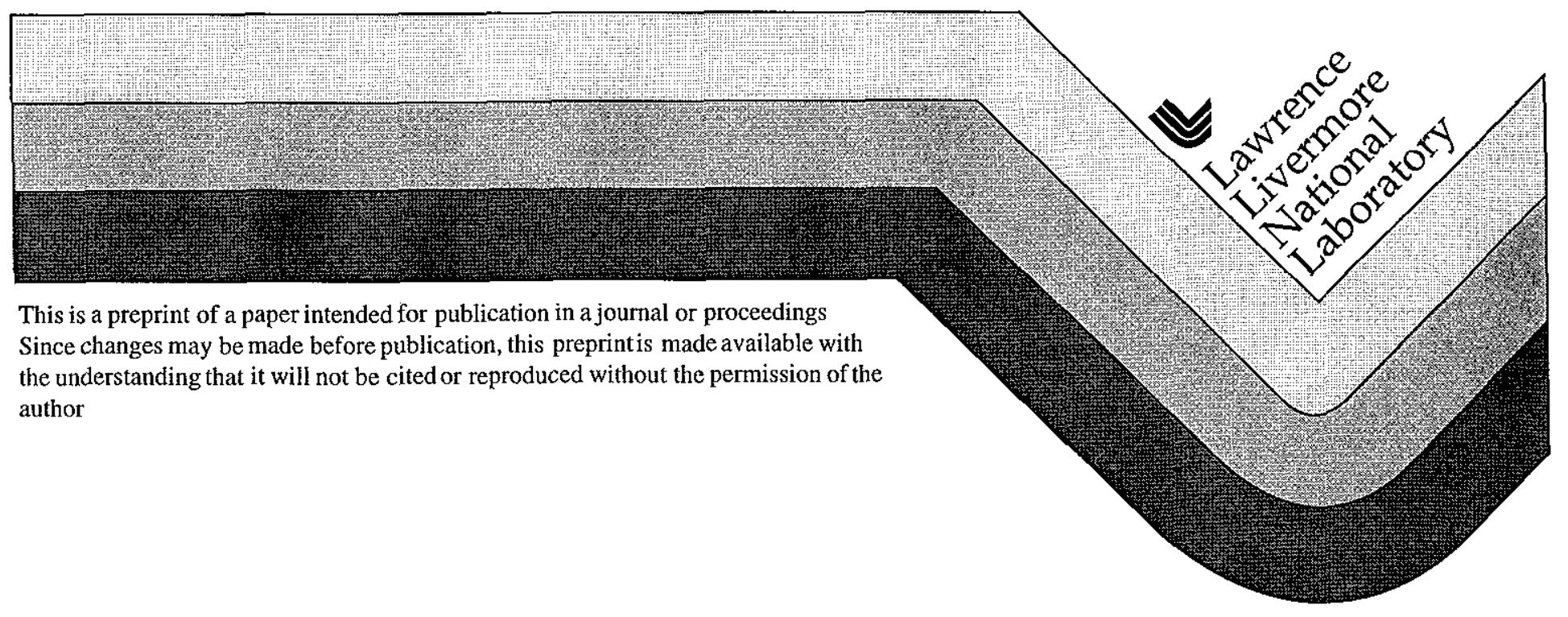




\section{DISCLAIMER}

This document was prepared as an account of work sponsored by an agency of the United States Government Neither the United States Government nor the University of California nor any of their employees, makes any warranty, express or implied, or assumes any legal liability or responsibility for the accuracy, completeness, or usefulness of any information, apparatus, product, or process disclosed, or represents that its use would not infringe privately owned rights Reference herein to any specific commercial product, process, or service by trade name, trademark, manufacturer, or otherwise, does not necessarily constitute or imply its endorsement, recommendation, or favoring by the United States Government or the University of California The views and opinions of authors expressed herein do not necessarily state or reflect those of the United States Government or the University of California, and shall not be used for advertising or product endorsement purposes 


\title{
Linear Dichroism and Resonant Photoemission in Gd
}

S.R. Mishra $^{1}$, T R. Cummins ${ }^{2}$, G.D. Waddill ${ }^{2}$, W.J. Gammon ${ }^{1}$, G. van der Laan ${ }^{3}, \mathrm{~K}$ W. Goodman ${ }^{4}$ and J.G. Tobin ${ }^{*} 4$

1 Department of Physics, Virginia Commonwealth Univ., Richmond, VA, USA, 2 Department of Physics, University of Missouri-Rolla, Rolla, MO, USA 65401, 3

Daresbury Laboratory, Warrington WA4 4AD, United Kingdom, 4 Lawrence Livermore National Laboratory, Livermore, CA, USA 94550.

*Corresponding Author Tele 925-422-7247, Fax 925-423-7040,

Email Tobin1@LLNL.Gov

\begin{abstract}
Magnetic Linear Dichroism in Angular Distributions (MLDAD) from Photoelectron Emission was used to probe the nature of Resonant Photoemission. Gd 5p and $\mathrm{Gd} 4 \mathrm{f}$ emission were investigated. Using novel theoretical simulations, we were able to show that temporal matching is a requirement for "True" Resonant Photoemission, where the Resonant Photoemission retains the characteristics of Photoelectron Emission.
\end{abstract}




\section{Discussion}

Resonant Photoemission is the phenemonon where the emission of $4 \mathrm{f}$ and $5 \mathrm{p}$ electrons from rare-earth metals and their compounds is strongly enhanced because a second emission channel opens up, e.g the photon has just enough energy to excite a $4 \mathrm{~d}$ electron to an unoccupied $4 \mathrm{f}$ level. In a generic picture, the indirect channel of the resonant photoemission is interpreted as due to a process where a $4 \mathrm{~d}$ electron in the initial state is first excited to the unoccupied $4 \mathrm{f}$ level, forming a tightly coupled, bound intermediate state, $4 \mathrm{~d}$ core hole plus $4 \mathrm{f}$ electrons. Then a decay via autoionization occurs into the final state, thus producing a final state identical to that obtained by a direct photoemission process for the ejected electron. [1] The transition rate is greatly enhanced if the excited state decay is by a Coster-Kronig or a super-Coster-Kronig [ (s)CK] process $[2,3]$ The key question is whether these processes are coherent or incoherent: Is it truly "resonant photoemission" or merely the incoherent addition of a second emission channel? Should the overall intensity be treated as a squaring of the sum of the amplitudes (coherent) or summing of the squares of the amplitudes (incoherent)? A true "resonant photoemission" process should be coherent, involving interference terms between the direct photoemission and indirect photoemission channels Possibly, incoherence would give rise to the loss of photoemission characteristics in the process, with a domination of auger-like properties

To this problem we have applied the new photoelectron spectroscopy technique of magnetic linear dichroism in angular distributions (MLDAD). [4-7] This technique is related to but distinct from the techniques of magnetic $\mathrm{x}$ ray circular dichroism (MXCD) in photoelectron spectroscopy and $x$ ray absorption. [14] The key is that while large dichroic effects in ferromagnets can be observed with MXCD-photoemission and MXCDabsorption, the large MLDAD effects in ferromagnets is solely a photoemission, not an absorption-driven, process. This is because the chirality which gives rise to magnetic sensitivity is due to the vectorial configuration in MLDAD as opposed to the intrinsic 
chirality of circularly polarized $\mathrm{x}$ rays in the MXCD techniques. In absorption, where there is an essential averaging over all emission angles, the vectorial chirality is lost. Thus, MLDAD is the ideal measurement to distinguish between photoemission and absorption processes. Angle-resolved photoemission in a magnetic system should show an MLDAD effect: $x$ ray absorption and thus auger-like emission will show no MLDAD effect. Experimantal details can be found elsewhere. [15-19] Theoretical spectra were calculated in intermediate coupling using Cowan's relativistic Hartree-Fock code.[20] Radiative transitions were taken into account to first order and (s)CK transitions to infinite order. [2123] Line broadening of the photoelectron state and experimental resolution were included by a convolution with a Lorentzian and a Gaussian, respectively. For the $4 \mathrm{f}$ emission the interference effects between the different photoemission final states and between direct and resonant channel were fully included For the 5 p emission the interference between the direct and resonant channel was treated as scalar in order to reduce the overall size of the calculation The interference term was excluded in the $5 \mathrm{p}$ calculation

The study included an extensive therotetical and experimental data set, collected "on" and "off" resonance Some of the spectra can be seen in Figure 1. These spectra, coupled to results not shown here, lead to the following conclusions The $\mathrm{Gd} 4 \mathrm{f}$ resonant photoemission is confirmed to be photoemission-like, because it shows an MLDAD effect on resonance. The Gd $5 p$ resonant emission is shown to be dominated by augerlike contributions, owing to the absence of an MXLD effect at resonance. The experiment results are confirmed by the theoretical simulations. It appears that temporal channel matching is a requirement for channel interference and the persistence of photoemission effects The Coster-Kronig decay that occurs in the $5 p$ emission occurs on a time scale of about $10^{-15}$ sec. [2] The super-Coster-Kronig delay of the $4 \mathrm{f}$ should be significantly faster [2,3] This would speed up the indirect channel, bringing it nearer to the time duration of $x$ ray absorption $\left(10^{-17} \mathrm{sec}\right.$ or less) that dominates the direct photoemission 
channel. Thus, not only must the energies of the two channels match but also the time duration, in order to observe "true resonant photoemission".

Figure 1. A series of experimental and theoretical $4 \mathrm{f}$ and $5 \mathrm{p}$ photoemission spectra (for the two opposite magnetization directions) and normalized difference curves. The photon energy was $150 \mathrm{eV}$ for the $4 \mathrm{f}$ and $151 \mathrm{eV}$ for the $5 \mathrm{p}$ (a) $\mathrm{Gd} 4 \mathrm{f}$, photoelectron spectra, experimental.

(b) Gd 4f, photoelectron spectra difference, experimental

(c) Gd 4f, photoelectron spectra, theory.

(d) Gd 4f, photoelectron spectra difference, theory.

(e) Gd 5p, photoelectron spectra, experimental

(f) Gd 5p, photoelectron spectra difference, experimental.

(g) Gd 5p, photoelectron spectra, theory.

(h) Gd 5p, photoelectron spectra difference, theory. EDC is energy distribution curve. The spectra in (a), (c), (e), \& (g) are EDC's, where the photon energy is held constant and the kinetic energy is scanned. PND stands for peak normalized difference, where the dichroism difference at each binding energy is divided by the sum of the two intensity maxima, one from each pair The photon energies of $150 \mathrm{eV}$ and $151 \mathrm{eV}$ are "on" resonance The relative intensities of the experimental curves were determined by normalizing to the valence band intensities and then correcting for the valence band cross sections.
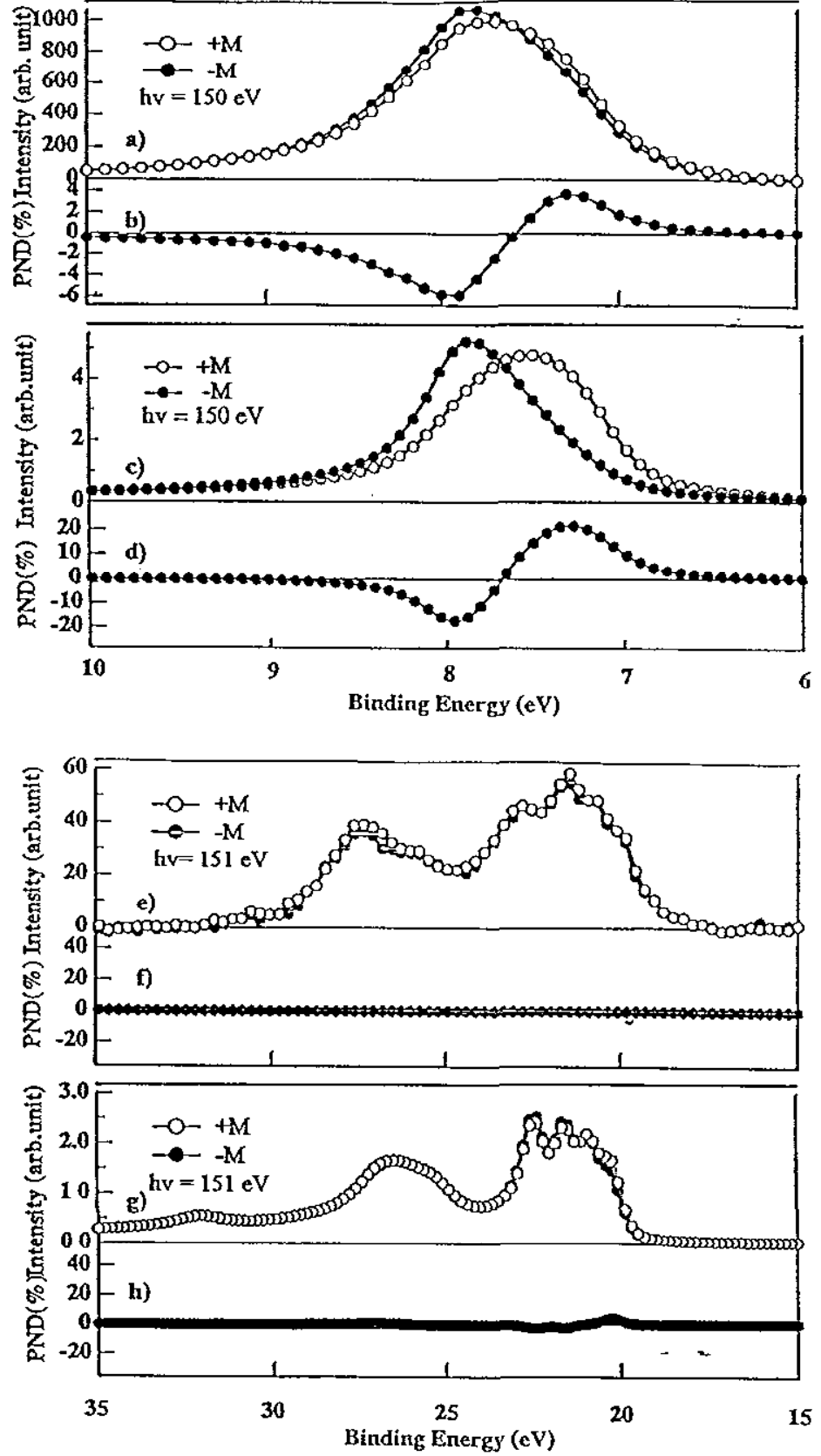


\section{Acknowledgements}

The authors, particularly JGT, would like to thank David Pappas for his guidance and aid, especially in the thesis work of W J. Gammon This work was performed under the auspices of the U.S Department of Energy by Lawrence Livermore National Laboratory under contract no. W-7405-Eng-48 Experiments were carried out at the Spectromicroscopy Facility (Beamline 7.0) at the Advance Light Source, built and supported by the U.S. Department of Energy. We would also like to thank F.O.

Schumann and R.F. Willis for aid in some of the data collection.

\section{References}

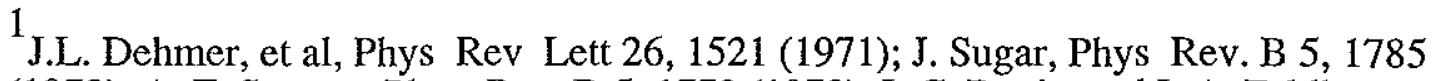
(1972), A F Starace, Phys. Rev. B 5, 1773 (1972), L C. Davis, and L A Feldkamp, Phys Rev. A 17, 2012 (1978), F. Gerken, et al Phys. Rev. Lett 47, 993 (1981); Extensive references by $\mathrm{J}$ Allen in Synchrotron Radiation Research, ed. R.Z. Bachrach (Plenum Press, New York, 1992), Vol. 1, p 253, Giant Resonances in Atoms, Molecules, and Solids, eds. J.P Connerade, J -M Esteva, and R.C. Karnatak, NATO ASI series B (Plenum Press, New York, 1987).

2

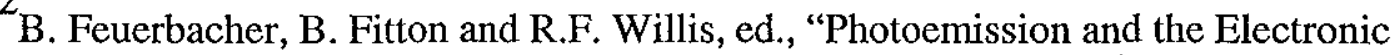
Properties of Surfaces", John Wiley \& Sons, New York, See Fig. 51 on Page 115.

3

T A. Carlson, "Photoemission and Auger Spectroscopy," Plenum Press, New York.

$$
4
$$

Ch. Roth, et al, Phys Rev. Lett 70, 3479 (1993), Solid State Commun 86647 (1993);

F.U. Hillebrecht, et al, Phys Rev B53, 12182 (1996)

5F Sirotti and G. Rossi, Phys Rev. B 49, 15682 (1994), G. Rossi, et al, Solid State Commun 90, 557 (1994)

6 W. Kuch, et al, Phys Rev. B $\underline{51}, 609$ (1995)

7 F.O Schumann, et al, Phys Rev. Lett $\underline{79}, 5166$ (1997)

${ }^{8}$ L Baumgarten, et al, Phys. Rev. Lett. 65, 492 (1990)

9 J.G. Tobin, et al, Phys. Rev. Lett $\underline{68}, 3642$ (1992).

10 C M. Schneider, et al, Phys. Rev. B 45, 5041 (1992). 
${ }^{11}$ B.T. Thole and G van der Laan, Phys. Rev. B 44, 12424 (1991)

${ }^{12}$ K. Starke, et al, Phys. Rev. B 48, 1329 (1993)

13 K. Starke, et al, Phys Rev. B 55, 2672 (1997); E. Arenholz, Ph D. Thesis, "Magnetic Dichroism in Photoemission from Lanthanide Materials: Basic Concepts and Applications", Wissenschaft and Technik Verlag, Berlin, 1996

${ }^{14}$ J.G Tobin, et al, Surf Sci. Lett. $\underline{395}, 227$ (1998)

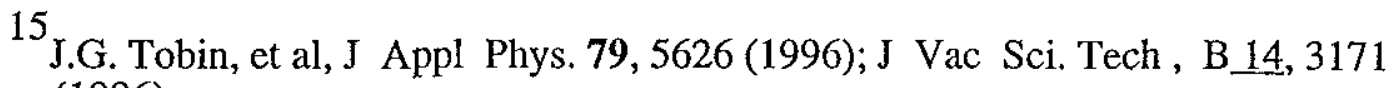
(1996).

16 J Denlinger et al Rev. Sci Instrum 66, 1342 (1995)

${ }^{17}$ W J Gammon, et al, J Vac. Sci. Technol A 15, 1 (1997)

${ }^{18}$ S.R. Mishra, et al, J. Vac. Sci Tech A 16, XXXX (1998)

${ }^{19}$ W.J. Gammon, M S Thesis, Virginia Commonwealth Univ., 1994, Unpublished.

20 R.D. Cowan, The Theory of Atomic Structure and Spectra, University of California Press, Berkeley (1981).

${ }^{21}$ G. van der Laan, et al, Phys Rev. B 46, 9336 (1992)

${ }^{22}$ G. Van der Laan, Phys Rev B 51, 240 (1995).

${ }^{23}$ G. van der Laan, et al, Phys. Rev. B 56, 3244 (1997) 


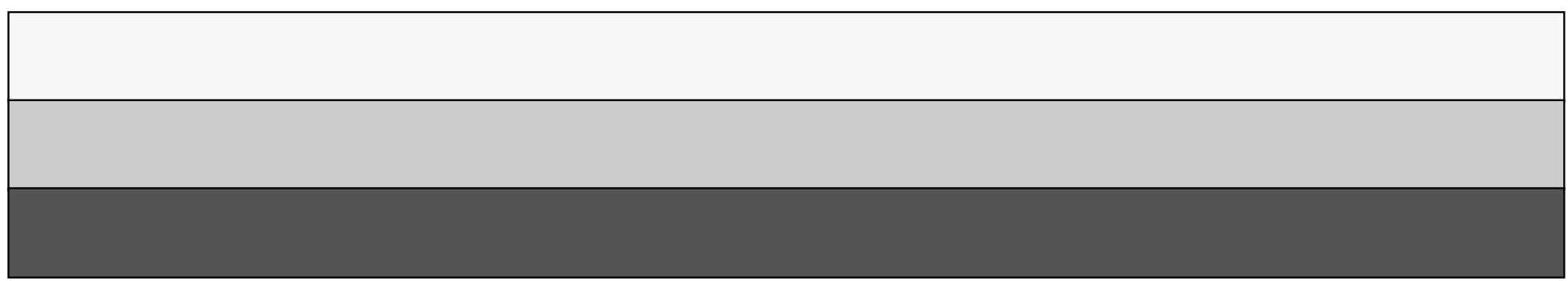

